\title{
Análise espacial de indicadores integrados determinantes da mortalidade por diarreia aguda em crianças menores de 1 ano em regiões geográficas
}

\author{
Spatial analysis of integrated determinant indicators \\ of mortality from acute diarrhea in children \\ under 1 year of age in geographical regions
}

Helena Ferraz Bühler ${ }^{1}$

Eliane Ignotti ${ }^{2}$

Sandra Mara Alves da Silva Neves ${ }^{3}$

Sandra Souza Hacon ${ }^{4}$

${ }^{1}$ Departamento de Enfermagem, Universidade do Estado de Mato Grosso, Campus de Diamantino.

R. Rui Barbosa 535, Jardim Eldorado. 78.400000 Diamantino MT Brasil.helenaferraz24@ hotmail.com

${ }^{2}$ Departamento de Enfermagem, Universidade do Estado de Mato Grosso, Campus de Cáceres.

${ }^{3}$ Departamento de Geografia, Universidade do Estado de Mato Grosso.

${ }^{4}$ Escola Nacional de Saúde Pública, Fiocruz.

\begin{abstract}
The scope of this study is to perform spatial analysis of integrated environmental and health indicators related to the factors affecting mortality due to diarrhea in children under 1 year of age in Brazilian regions in 2010. Seven environmental indicators, compiled from the IBGE System for Automatic Recovery of the Population Census 2010 database, were formulated. The data with respect to deaths due to diarrhea in children under 1 year of age and live births were obtained from the databases of the Mortality Information Systems and the Live Births Information System of the IT Department of the Unified Health System. The microregions located in the North and Northeast regions revealed 5 and 4 times the rate of mortality in 2009, respectively, due to diarrhea in children under 1 year of age than the Southern Region. Children under 1 year of age living in the microregions located in the North and Northeast are more exposed to risk of death from diarrhea, since the worst figures for the environmental indicators related to poverty and sanitation are concentrated in these locations. In this sense, social, economic, environmental, cultural and health public policies should be based on the principle of equity to address the different local needs of each region.
\end{abstract}

Key words Death, Infant, Diarrhea, Environmental factors
Resumo O objetivo deste estudo é realizar a análise espacial de indicadores integrados de ambiente e saúde relativos aos fatores condicionantes da mortalidade por diarreia em menores de 1 ano nas regiões brasileiras no ano de 2010. Foram formulados 7 indicadores socioambientais, construídos a partir do banco de dados do Sistema IBGE de Recuperação Automática do Censo Demográfico de 2010. Os dados de óbitos por diarreia em crianças menores de 1 ano e de nascidos vivos foram obtidos das bases de dados dos Sistemas de Informação de Mortalidade e do Sistema de Informação de Nascidos Vivos do Departamento de Informática do Sistema Único de Saúde. As microrregiões situadas nas regiões Norte e Nordeste, apresentaram, em 2009, respectivamente, 5 e 4 vezes mais taxa de mortalidade por diarreia em menores de 1 ano, que a região Sul. As crianças menores de 1 ano residentes nas microrregiões localizadas nas regiões Norte e Nordeste estão mais expostas ao risco de óbito pela diarreia, uma vez que nestes locais concentram-se os piores valores para os indicadores socioambientais relacionados à pobreza e ao saneamento básico. Neste sentido, políticas públicas sociais, econômicas, ambientais, culturais e de saúde devem embasar-se no princípio de equidade para atender as diferentes necessidades locais de cada região.

Palavras-chave Morte, Infantil, Diarreia, Fatores ambientais 


\section{Introdução}

As doenças diarreicas agudas infecciosas e transmissíveis são provocadas por diferentes agentes enteropatógenos, sendo os principais as bactérias, os vírus e os protozoários. Os efeitos fisiológicos mais importantes são desidratação e desnutrição que dificultam o ganho de peso e altura nas crianças, e pode ocasionar retardo no intelecto infantil ${ }^{1-4}$.

Ainda que a progressão da evolução clínica aguda e fatal do processo saúde-doença na população infantil seja determinada por múltiplas causas, enfatiza-se que a pobreza é um importante aspecto que prediz as condições de saúde na infância ${ }^{5,6}$. A morbimortalidade por diarreia infantil está condicionada principalmente ao baixo nível socioeconômico da população, sendo este um dos principais fatores, que influencia as condições de saneamento básico precário e comportamento higiênico pessoal e doméstico insatisfatório ${ }^{7-11}$.

Estima-se que aproximadamente 10,5 milhões de crianças menores de 05 anos morrem todo ano nos países mais pobres, por doenças infecciosas e parasitárias, principalmente pneumonia, diarreia, sarampo e AIDS/HIV ${ }^{12-14}$.

As regiões brasileiras mostram diferentes condições demográficas, econômicas, sociais, culturais e de saúde. A região Norte possui a maior parte da Floresta Amazônica, tem a menor densidade populacional (3,9 pessoas por $\left.\mathrm{km}^{2}\right)$ e é a segunda região mais pobre do País, depois da Nordeste, com elevada proporção de residências sem coleta de lixo e com esgotamento sanitário a céu aberto ${ }^{15,16}$.

No Brasil, apesar de os dados oficiais apontarem para a queda da mortalidade em menores de 05 anos, as regiões Norte e Nordeste concentram a maioria dos óbitos ${ }^{17}$. As taxas de mortalidade por diarreia infantil no Brasil mostram que os menores de 1 ano são os mais vulneráveis e as regiões Norte e Nordeste lideram as taxas mais elevadas de óbito também nesta faixa etária ${ }^{18}$.

Este estudo epidemiológico ambiental teve por objetivo a análise espacial de indicadores integrados de ambiente e saúde relativos aos fatores condicionantes da mortalidade por diarreia em menores de 1 ano nas regiões brasileiras no ano de 2010 .

\section{Material e métodos}

\section{Desenho do estudo e levantamento de dados secundários}

Estudo epidemiológico ambiental, com delineamento ecológico acerca da ocorrência da mortalidade por diarreia infantil nas microrregiões das regiões brasileiras, que se distribuem distintamente pelo território brasileiro.

O Brasil é constituído por 558 microrregiões, sendo que a região Nordeste apresenta 34\% das microrregiões, seguida da Sudeste com 27\%, Sul (17\%), Norte (11\%) e Centro-Oeste (9\%). Possui aproximadamente 3 milhões de crianças menores de 1 ano de idade, das quais a maior proporção encontra-se nas regiões Sudeste e Nordeste, seguidas das regiões Sul, Norte e Centro-Oeste ${ }^{15}$.

As crianças menores de 1 ano são mais suscetíveis ao quadro grave de diarreia, portanto com maior risco de óbito pelo agravo, o que determina a opção por estudar este grupo etário. A agregação dos dados municipais em microrregiões possibilita análises comparativas em toda área do estudo, e condiciona maior estabilidade das taxas.

Foram formulados 7 indicadores que caracterizaram determinantes da diarreia infantil no Brasil, baseados em uma revisão bibliográfica.

Os dados secundários foram obtidos segundo as microrregiões das regiões brasileiras no Banco de Dados Agregados do Instituto Brasileiro de Geografia e Estatística (SIDRA/IBGE) do Censo Demográfico de 2010.

Para a construção do indicador de percentual de moradores em extrema pobreza, utilizouse como numerador a população com classe de rendimento nominal mensal per capita até $1 / 8$ de salário mínimo (baseado no salário mínimo de $\mathrm{R} \$ 510,00)$, sem rendimento ou com rendimentos por algum benefício social. A razão de dependência foi representada no numerador pelo número de residentes de 0 a 14 anos e de 60 e mais anos de idade dividido pela população de 15 a 59 anos de idade. O número médio de moradores por domicílio foi obtido no banco de dados do IBGE. Os numeradores dos indicadores do percentual de moradores com esgotamento sanitário por vala ou outro tipo e sem banheiro e sanitário foram formulados considerando o número de moradores que residem em domicílio com essas características. Também se utilizou como numeradores para os indicadores de percentual de domicílios sem água canalizada dentro de casa, o número de domicílios sem água canalizada dentro de casa e 
percentual de moradores sem coleta de lixo, os moradores que queimam, enterram, jogam lixo em terreno baldio, rio, lago ou mar ou aplicam outro destino ao lixo domiciliar. Exceto para razão de dependência e média de moradores por domicílio, os denominadores dos indicadores correspondem ao total de moradores residentes nas microrregiões das regiões brasileiras.

Os dados de óbitos por diarreia em crianças menores de 1 ano e de nascidos vivos, que constituem o numerador e denominador, respectivamente, do indicador de saúde desta pesquisa foi obtido do site do Departamento de Informática do Sistema Único de Saúde (DATASUS), sendo consultadas as bases de dados dos Sistemas de Informação de Mortalidade (SIM) e do Sistema de Informação de Nascidos Vivos (SINASC). Os dados de mortalidade correspondem aos óbitos associados às gastroenterites e diarreias segundo a 10a Revisão da Classificação Internacional de Doenças (CID - 10) da Organização Mundial de Saúde - Capítulo I, códigos A00 a A09, delimitando a faixa etária menores de 1 ano, e o ano de 2009.

\section{Análises dos dados}

Para a seleção dos indicadores utilizados na construção daqueles integrados de ambiente e saúde foi realizada a análise de correlação de Spearman, sendo selecionados os com correlações positivas, com nível de significância $\leq 0,20$, existentes entre os indicadores de ambiente e o desfecho epidemiológico do estudo.

Para as microrregiões da região Norte, foram selecionados todos os indicadores socioambientais testados para integrar ao desfecho de mortalidade por diarreia infantil. Para a região Nordeste selecionaram-se os indicadores: percentual de moradores em extrema pobreza, razão de dependência, média de moradores por domicilio, percentual de moradores com esgotamento sanitário do tipo vala ou outro tipo, sem banheiro e sanitário, e domicílios sem água canalizada na residência. Para as microrregiões da região Centro-Oeste selecionou-se: o percentual de moradores em extrema pobreza, razão de dependência e média de moradores por domicilio. Selecionou-se apenas o percentual de moradores em extrema pobreza para integrar a mortalidade por diarreia em menores de 1 ano nas microrregiões situadas na região Sudeste. Enfatiza-se que para as microrregiões na região Sul não foi construído indicador integrado de ambiente e saúde, devido à ausência de significância na correlação entre os indicadores testados (Tabela 1).

Foi realizada análise estatística de regressão linear múltipla para estabelecer pesos aos indicadores de ambiente, para a integração ao indicador de saúde. As variáveis independentes $(\mathrm{x})$ foram os indicadores ambientais, e a variável dependente (y) foi o indicador de saúde. Esta análise se baseou na inserção das variáveis independentes no modelo (indicadores de ambiente selecionados pela correlação), e observação da significância ao nível de $5 \%$ de cada uma das variáveis. As variáveis independentes não significantes ou que não serviram para ajuste dos modelos foram excluídas da análise. Os valores significativos a partir da regressão linear múltipla compõe o modelo explicativo da diarreia infantil nas microrregiões das regiões brasileiras (Tabela 2).

Para as análises foi utilizado o programa computacional $\mathrm{R}$ versão 2.15.2.

Para a integração dos indicadores de ambiente ao de saúde, que foram selecionados previamente por meio das correlações e ponderados pela regressão múltipla, os mesmos foram trans-

Tabela 1. Matriz de correlação de Spearman entre as variáveis independentes e taxa de mortalidade por diarreia em menores de 1 ano segundo microrregiões. Regiões geográficas brasileiras, 2010.

\begin{tabular}{lccccc}
\hline \multirow{2}{*}{\multicolumn{1}{c}{ Variáveis independentes }} & \multicolumn{5}{c}{ Coeficiente de correlação ( $\rho$ valor) } \\
\cline { 2 - 6 } & NMDDA < 1 \\
\hline PMEP & Norte & Nordeste & Centro-Oeste & Sudeste & Sul \\
RD &, $38(, 002)$ &, $21(, 004)$ &, $22(, 117)$ &, $14(, 078)$ &, $12(, 228)$ \\
MMD &, $29(, 019)$ &, $19(, 008)$ &, $21(, 135)$ &, $03(, 682)$ &, $01(, 893)$ \\
PMESVO &, $32(, 009)$ &, $18(, 012)$ &, $24(, 082)$ &, $05(, 510)$ &,$- 00(, 963)$ \\
PMSBS &, $34(, 005)$ &, $17(, 019)$ &, $14(, 310)$ &, $07(, 376)$ &, $01(, 853)$ \\
PDSAC &, $29(, 017)$ &, $13(, 065)$ &, $09(, 486)$ &,$- 00(, 995)$ &, $08(, 409)$ \\
PMSCL &, $44(, 000)$ &, $09(, 192)$ &, $21(, 119)$ &, $07(, 365)$ &, $04(, 639)$ \\
&, $30(, 015)$ &, $03(, 657)$ &, $15(, 272)$ &,$- 05(, 513)$ &,$- 01(, 896)$ \\
\hline
\end{tabular}




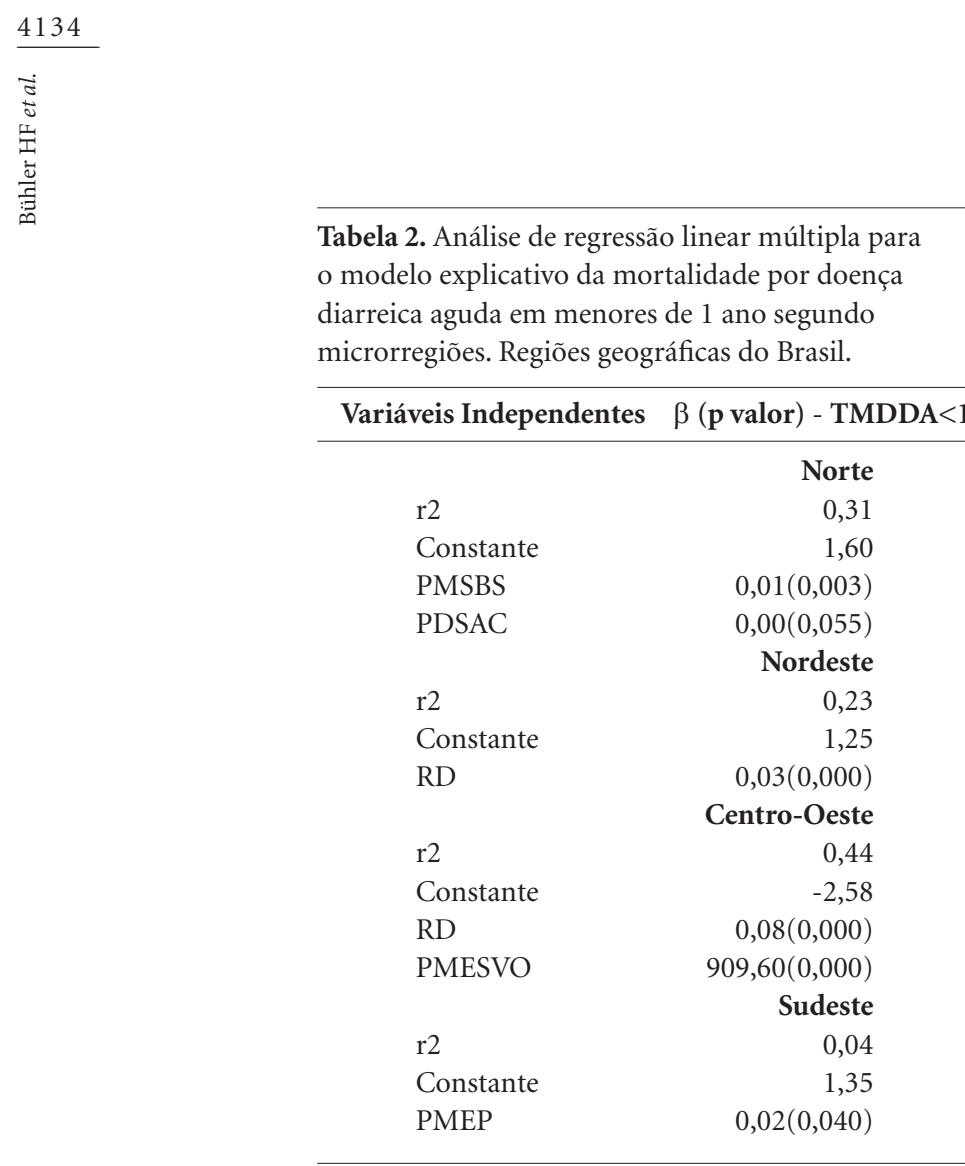

formados em índices, com valores variando entre 0 e 1, sendo considerado pior o de menor valor, e melhor o de valor maior. Posteriormente, os índices ambientais foram agregados ao desfecho do estudo. Para esta etapa utilizou-se a técnica estatística de média aritmética simples e ponderada, formando o indicador integrado de ambiente e saúde para as microrregiões nas regiões brasileiras ${ }^{19}$.

Os indicadores integrados foram estratificados em quartis, sendo que cada $1 / 4$ dos intervalos foi categorizado em muito bom, bom, ruim e péssimo.

Para explorar a significância da correlação espacial dos indicadores integrados de ambiente e saúde foi realizada a análise de indicadores locais de autocorrelação espacial, sendo gerados os mapas Moran Map, que mostra os seguintes parâmetros:

- Q1 e Q2: indicam pontos de associação espacial positiva, no sentido de que uma localização possui vizinhos com valores semelhantes, categorizados como Q1(alto-alto) e Q2 (baixo -baixo);

- Q3 e Q4: indicam pontos de associação espacial negativa, no sentido de que uma localização possui vizinhos com valores distintos, categorizados como Q3 (baixo-alto) e Q4 (alto -baixo).
Para efeito de análise, foi utilizada a malha digital das Unidades Federativas de cada uma das regiões brasileiras disponibilizada no site do IBGE.

O software utilizado para análise espacial foi o ArcGIS versão 10.1 da Esri.

\section{Resultados}

As microrregiões com maiores taxas de mortalidade por doença diarreica aguda em menores de 1 ano em 2009 estão situadas nas regiões Norte e o Nordeste, que apresentaram, respectivamente, 5 e 4 vezes mais taxa de mortalidade por diarreia em menores de 1 ano que a região Sul (Figura 1).

Para as microrregiões da região Norte, as localizadas a oeste, nos estados de Amazonas e Acre, observam-se maior parte de microrregiões com indicadores agregados à mortalidade por diarreia infantil apresentando valores categorizados como péssimos e ruins. Exceto as microrregiões situadas nas regiões Metropolitanas de Manaus e Belém, que mostram valores ruins, o estado do Pará apresenta maior parte de microrregiões com valores bons e muito bons, que também são encontrados nas microrregiões dos estados de Tocantins e Rondônia. Nas microrregiões da região Nordeste, observa-se na porção noroeste e centro-sul (estados do Maranhão, Ceará, Piauí e Pernambuco) e porção norte do estado da Bahia valores categorizados como péssimos e ruins. Por outro lado, a porção litorânea apresentou maior frequência de indicadores bons e muito bons. Para as microrregiões da região Centro-Oeste, observa-se nos estados de Mato Grosso e Mato Grosso do Sul, valores nos quartis péssimos e ruins com maior frequência, seguidos do estado de Goiás. O Distrito Federal destaca-se pela predominância de frequência de dados muito bons para a análise do efeito de mortalidade por diarreia em menores de 1 ano. Para as microrregiões na região Sudeste, observam-se na distribuição espacial valores péssimos e ruins predominando na porção norte da região Sudeste, onde se situam a área metropolitana de Belo Horizonte e Vale do Aço do estado de Minas Gerais. As microrregiões situadas no estado de Minas Gerais correspondem ao Vale do Jequitinhonha, área extremamente pobre, constituído por 80 municípios, ocupando 15\% do Estado (Figuras 2A, 2B, 2C e 2D).

Para a região Norte, observa-se que os valores Q2 que indicam dependência espacial significante estão em maior parte nas microrregiões dos 
estados do Acre e Amazonas, e na porção oeste do estado do Pará. Observa-se na região Nordeste que os padrões de dependência espacial em Q2 significantes para os indicadores integrados para mortalidade por diarreia em menores de 1 ano concentram-se nas microrregiões situadas no estado do Maranhão. Para a região Centro-Oeste, observa-se que a significância dos valores em Q2 para os indicadores integrados de ambiente e saúde para mortalidade, foi estatisticamente sig-

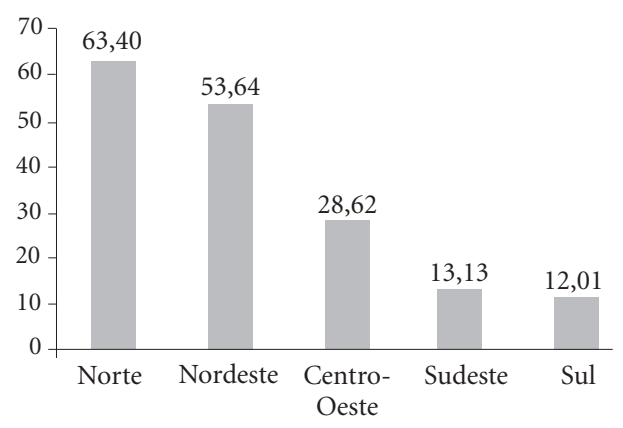

Figura 1. Taxa de mortalidade por diarreia em crianças menores de 1 ano segundo regiões geográficas brasileiras, 2009. nificante na microrregião de Bodoquena, que se localiza na porção oeste no estado de Mato Grosso do Sul. As microrregiões da região Sudeste foram estatisticamente significante em Q2 para as localizadas na porção norte do estado de Minas Gerais (Figuras 3A, 3B, 3C e 3D).

\section{Discussão}

As crianças menores de 1 ano residentes nas microrregiões situadas nas regiões Norte e Nordeste do Brasil morrem mais por doença diarreica aguda se comparadas a aquelas residentes nas outras regiões brasileiras e principalmente com relação à região Sul do País.

Dentre os indicadores associados aos óbitos por diarreia infantil nas microrregiões brasileiras, o de extrema pobreza destacou-se por obter correlação com todas as regiões, exceto a região Sul. Para as microrregiões situadas nas regiões Norte e Nordeste, os principais indicadores foram os relacionados às condições sociais e demográficas, e ao saneamento básico. Para as microrregiões situadas nas regiões Centro-Oeste e Sudeste, os indicadores sociodemográficos mostraram-se significantes com os óbitos por diarreia infantil.
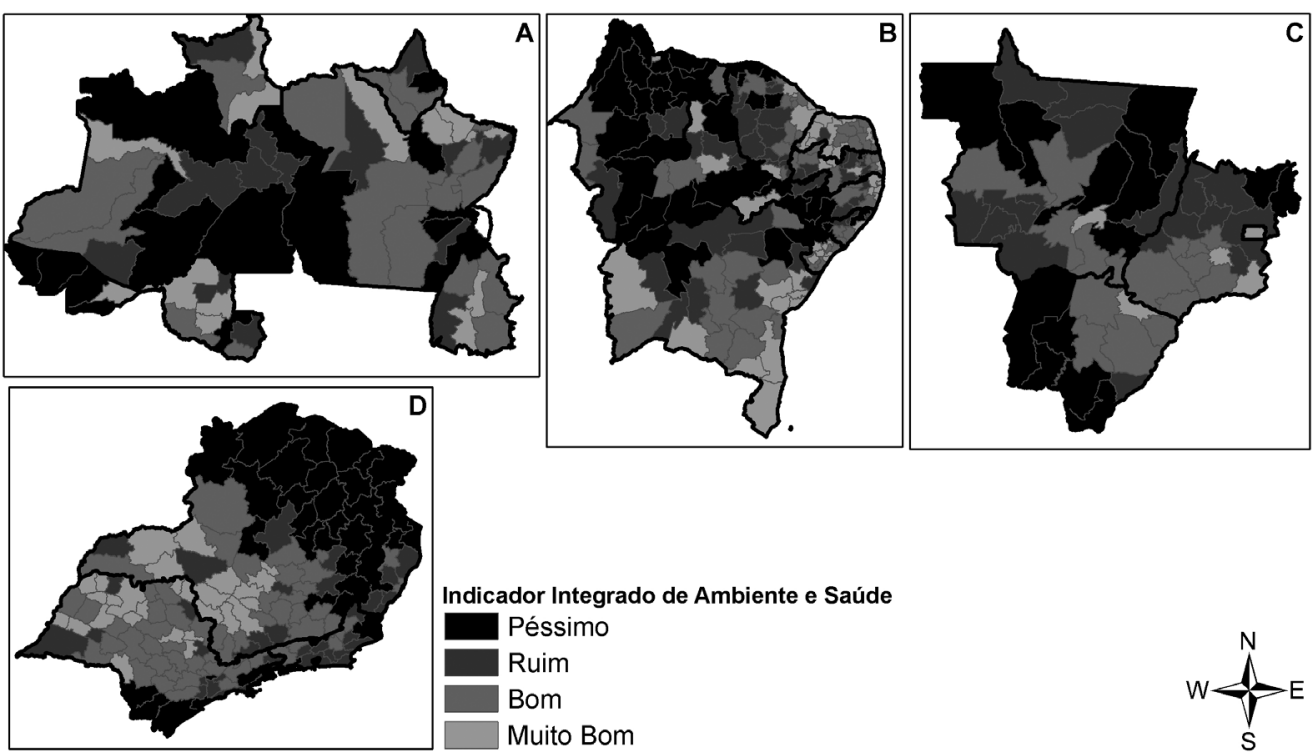

Figura 2. Distribuição espacial dos indicadores integrados de ambiente e saúde para mortalidade por diarreia em menores de 1 ano segundo microrregiões brasileiras. Regiões Norte (A), Nordeste (B), Centro-Oeste (C) e Sudeste (D). Brasil, 2010/2009. 


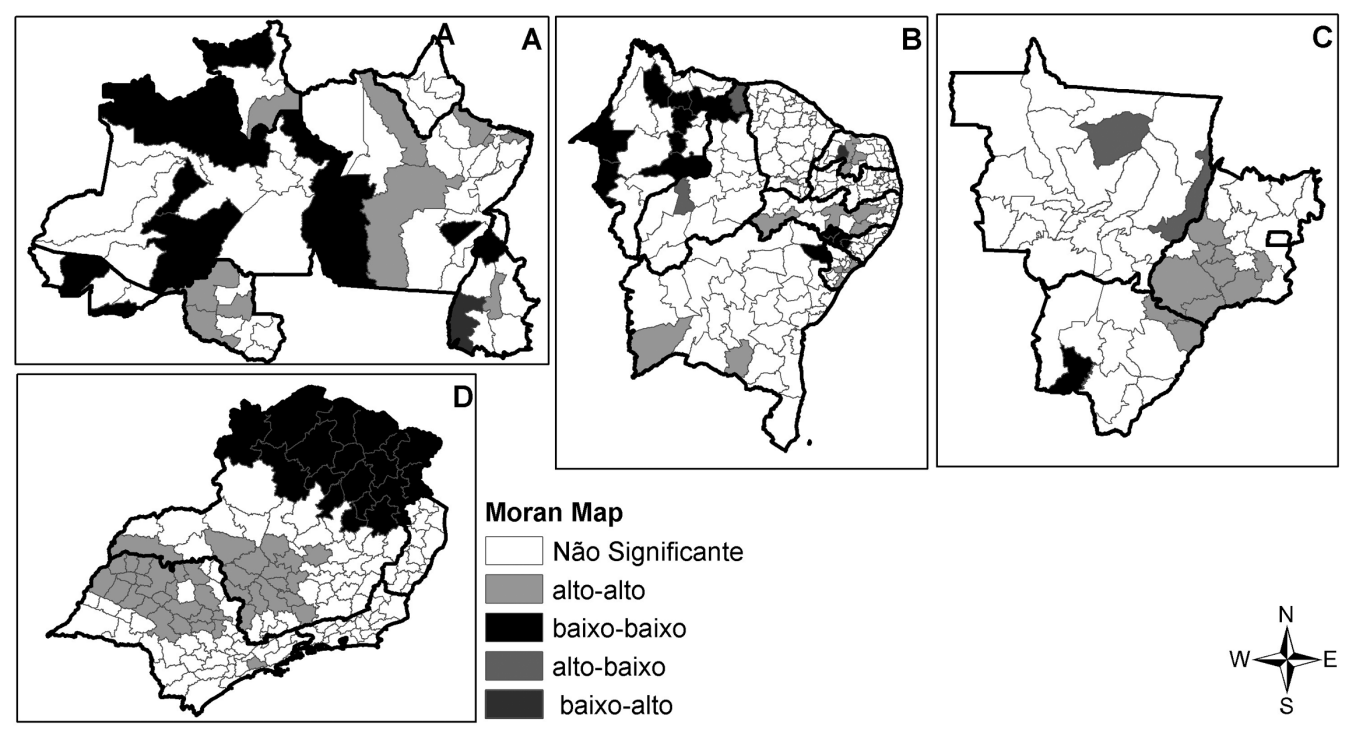

Figura 3. Estatística espacial dos indicadores integrados de ambiente e saúde para mortalidade por diarreia em menores de 1 ano segundo microrregiões brasileiras. Regiões Norte (A), Nordeste (B), Centro-Oeste (C) e Sudeste (D). Brasil, 2010/2009.

No Brasil, os indicadores básicos do País mostram disparidades regionais com relação à taxa de mortalidade infantil, que apresenta tendência descendente há mais de 10 anos. As regiões Norte e Nordeste mostram as maiores taxas, e em torno de $80 \%$ dos óbitos por diarreia foram em menores de 1 ano no período de 2000 a $2010^{17,18,20}$.

Neste estudo o indicador de mortalidade por diarreia infantil apresentou comportamento distinto entre as microrregiões nas regiões brasileiras, com destaque para aquelas situadas no Norte e Nordeste, que apresentaram maior frequência dos agravos estudados.

Tal achado é condizente com outras publicações, uma vez que os municípios situados nos estados do Pará, Piauí, Paraíba, Pernambuco e Bahia destacaram-se pelas elevadas taxas de internações e óbitos por diarreia em menores de 05 anos. Para as capitais Boa Vista, Belém, Teresina, Recife, Aracajú e Manaus, as taxas de internações por diarreia em menores de 05 anos, mostraram tendências de elevação na década de 1995 a $2005^{21-23}$.

Ainda que os estudos citados tenham abordado faixa etária e escala de análise diferente do presente estudo, os resultados desta pesquisa refletem a coerência de análises ecológicas para diarreia, apontando no intervalo de faixa etária para menores de 05 anos, que os menores de 1 ano são mais vulneráveis e porções territoriais com riscos mais elevados semelhantes ao que já foi divulgado, ou seja, as regiões Norte e Nordeste.

Outra questão que deve ser considerada com relação às taxas elevadas de mortalidade por diarreia em menores de 1 ano nas microrregiões situadas nas regiões Norte, Nordeste e Centro-Oeste é o elevado percentual de população indígena principalmente na área rural destas porções territoriais do País.

Dentre as causas de internações em crianças indígenas menores de 05 anos de idade, as diarreias e infecções respiratórias são as principais ${ }^{24}$. A população que se autodeclara indígena cresceu aproximadamente $11 \%$ na última década e a região Norte concentra 37,4\% dos povos indígenas e $43 \%$ das crianças indígenas menores de 05 anos do País. Entre as Unidades Federativas, o estado do Amazonas apresenta o maior percentual do País, com 20\% da população total de índios ${ }^{24,25}$.

A análise espacial para os indicadores integrados nas microrregiões da região Norte mostrou que a maior frequência de valores categorizados como ruim e péssimo, e valores Q2 significativos concentram-se no estado do Amazonas.

Tal achado já foi identificado na literatura, com relação à prevalência de parasitoses intestinais associadas com fatores socioambientais em áreas periféricas urbanas na cidade de Manaus, 
foi encontrado como fatores de risco para sua prevalência a ausência de saneamento básico, representado por abastecimento de água por cacimba e esgotamento sanitário a céu aberto ${ }^{26}$.

Para as microrregiões na região Centro-Oeste pode ser levantada a mesma discussão com relação à população indígena, uma vez que na distribuição espacial as microrregiões com valores categorizados como péssimos e ruins no estado de Mato Grosso envolvem os municípios com maior concentração de população indígena, o Parque Nacional do Xingu. Tal fato possivelmente influenciou a significância estatística para os valores Q2 para os indicadores integrados para mortalidade na microrregião do Médio Araguaia do estado de Mato Grosso.

O Parque Nacional do Xingu localiza-se na porção nordeste do estado de Mato Grosso e porção sul da Amazônia brasileira. As microrregiões de saúde do estado de Mato Grosso, denominadas Sul mato-grossense, Baixada Cuiabana, Médio e Baixo Araguaia compõe a rede de serviços de referências para atendimento hospitalares aos municípios do Parque Nacional do Xingu, e no estudo em tela, compõe os municípios das microrregiões com valores péssimos e ruins para os indicadores integrados para mortalidade por diarreia em menores de $1 \mathrm{ano}^{27}$.

A região Centro-Oeste possui o maior percentual de hospitalizações de crianças indígenas menores de 05 anos, sendo a diarreia a primeira causa de internação, relacionada às condições de saneamento precário, desmame precoce e contaminação dos alimentos da dieta infantil, permitindo a permanência do ciclo de transmissão fecal-oral ${ }^{24}$.

É evidente que nas regiões menos desenvolvidas os óbitos em decorrência da diarreia em crianças menores de 1 ano, sejam maiores, retratando sua relação com questões socioeconômicas e ambientais ${ }^{28}$. A ausência de correlação encontrada entre os indicadores demográficos e ambientais com taxa de internação (dados não apresentados), e desta com taxa de mortalidade por diarreia em menores de 1 ano nas microrregiões da região Norte pode relacionar-se à insuficiência de cobertura de unidades básicas de saúde em locais com piores condições de vida, que dificultam o acesso hospitalar a internações destas crianças, levando-as ao óbito sem receber assistência básica e hospitalar por este agravo.

Este achado permite ainda a reflexão acerca da procura do serviço de saúde pelo responsável quando a criança indígena encontra-se doente. $\mathrm{O}$ inquérito nacional acerca de saúde e nutrição dos povos indígenas no Brasil mostrou que na região Norte aproximadamente $30 \%$ das famílias não procurou ajuda no Posto de Saúde Indígena da aldeia ou equipe multidisciplinar de saúde indígena quando a criança desenvolveu quadro diarreico e infeccioso respiratório ${ }^{24}$. $\mathrm{O}$ aspecto cultural e o comportamento das famílias frente a situações do processo saúde-doença influencia o resultado de um desfecho agudo de saúde, obviamente tornando-se progressivamente mais grave, principalmente quando se trata de crianças e idosos.

Em relação aos indicadores que explicaram a ocorrência de mortes por diarreia infantil, as análises mostram que as microrregiões situadas nas regiões Norte, Nordeste e Centro-Oeste, exceto a razão de dependência, associam-se às questões de saneamento básico, sobretudo, esgotamento sanitário e abastecimento de água.

A permanência de doenças relacionadas ao saneamento ambiental inadequado nas macrorregiões Norte e Nordeste foi recentemente identificada nos municípios brasileiros por Fonseca e Vasconcelos $^{29}$.

Calijuri et al. ${ }^{30}$ ao analisarem através de estudo transversal em cidade do Norte do Brasil indicadores de saúde ambiental e de saneamento, mostraram que probabilidade de quadro diarreico em menores de 05 anos é maior em locais sujeitos a inundações, formados por invasões e ruas não pavimentadas que em relação às localidades com domicílios em ruas pavimentadas, com rede de drenagem e rede coletora de esgoto. O mesmo estudo também envolveu a caracterização dos domicílios, prevalecendo no primeiro local, aqueles com deficiências no abastecimento de água, inexistência de esgotamento e vasos sanitários nos banheiros das residências, com predominância de fossa seca e apenas 50\% de cobertura na coleta de lixo. Fonseca et al. ${ }^{31}$, encontraram em 10 municípios do Norte e Nordeste brasileiro, associações entre prevalência de Geo-helmintiases e renda familiar menor que 1 salário mínimo, presença de lixo próximo de casa, ausência de água encanada e número de residentes na moradia maior que 5 pessoas. Franco Netto et al..$^{32}$, reforçam os achados ao utilizar metodologia semelhante a este estudo para as Regiões e Unidades Federativas do Brasil com dados de 2000 a 2006. Os autores concluíram que entre os Estados, aqueles das regiões Norte e Nordeste concentram $53 \%$ da população extremamente pobre. Os estados do Maranhão e Piauí lideram os maiores percentuais para os domicílios sem coleta e tratamento de esgotamento sanitário, e os indicadores 
de saúde relacionados ao saneamento ambiental inadequado apresentaram-se fortemente correlacionados às dimensões socioambientais testadas.

Os estudos descritos utilizaram dados individuais, através de delineamentos transversais e ecológicos, mas com variáveis aproximadas aos indicadores ora construídos. Os resultados dos autores corroboram nossos resultados, uma vez que a renda familiar reflete as características de renda que no estudo fora representada pelo indicador de extrema pobreza. Ainda que no estudo a pobreza não foi significante na análise das microrregiões das regiões Norte e Nordeste, a distribuição espacial mostra que a população destas macrorregiões, é predominantemente pobre, e que, portanto, coerente com a análise estatística, que impõe maior importância para a mortalidade por diarreia infantil a questões ligadas à infraestrutura urbana e nos domicílios.

As condições sociais de vida desempenha importante determinante das condições de saúde da população, principalmente quando se trata da população mais pobre e infantil ${ }^{5,6}$. Portanto, políticas públicas que diminuem a desigualdade social são essenciais para o combate às iniquidades em saúde, diminuindo assim diferenciais de exposição a riscos, como a população infantil que vive em condições de habitação insalubres ${ }^{33}$, e que incrementam as mortes por diarreia nas regiões mais pobres do País, tais como Norte e Nordeste ${ }^{19}$.

Para as microrregiões situadas nas regiões mais desenvolvidas e analisadas no estudo, como Sudeste e Sul, apenas na região Sudeste a mortalidade por diarreia aguda em menores de 1 ano associou-se com o indicador de extrema pobreza. Isto por que na distribuição espacial é evidente que as microrregiões com melhores condições de infraestrutura estão concentradas nestas regiões do Brasil, caracterizando a pobreza o diferencial para indicadores de saúde da diarreia infantil nas microrregiões na região Sudeste. Estes achados possuem consistência científica na literatura, uma vez que Teixeira e Heller', em estudo transversal, concluíram que a elevada prevalência de diarreia infantil encontrada em assentamentos subnormais de uma cidade de porte médio do Sudeste brasileiro é comparável à encontrada na região Nordeste do Brasil. Tal fato deve-se à concentração da doença nas periferias e porções economicamente mais pobres das cidades de grande e médio porte brasileiras.
Especificamente nesta pesquisa, se utilizou a técnica de geoprocessamento de dados secundários de estatísticas vitais, sociodemográficos e ambientais dos sistemas de informação do País (DATASUS e IBGE), o que possibilitou a análise do contexto socioeconômico, ambiental e de saúde na área de estudo.

Conclui-se que a diarreia infantil apresenta-se como um agravo que mostra a iniquidade em saúde no território brasileiro. As crianças menores de 1 ano residentes nas microrregiões localizadas nas regiões Norte e Nordeste são as mais expostas ao risco de óbito pela diarreia, pois nestes locais concentram-se os piores valores para os indicadores socioambientais analisados, principalmente no que diz respeito à pobreza $\mathrm{e}$ ao saneamento básico.

Neste sentido, políticas públicas sociais, econômicas, ambientais, culturais e de saúde devem embasar-se no princípio de equidade para atender as diferentes necessidades locais de cada região ${ }^{34}$.

A análise estatística espacial solidificou e definiu mais precisamente as microrregiões que apresentam os piores valores para os indicadores integrados de ambiente e saúde nas regiões brasileiras. Os estados que apresentaram piores categorizações nos indicadores integrados de ambiente e saúde, são na região Norte, o Acre, o Amazonas e o Pará, região Nordeste, o Maranhão, o Piauí, a Bahia e o Ceará, na região Centro-Oeste, o Mato Grosso, o Mato Grosso do Sul e Goiás e na região Sudeste, Minas Gerais.

\section{Colaboradores}

HF Bühler trabalhou na concepção, redação do artigo, análise e interpretação dos resultados, E Ignotti no delineamento, análise e interpretação dos resultados, e revisão crítica, SMAS Neves e SS Hacon na interpretação dos resultados e revisão crítica. Todas as autoras participaram da aprovação da versão a ser publicada. 


\section{Referências}

1. Motta MEFA, Silva GAP. Diarreia por parasitas. Rev. Bras. Saude Mater. Infant. [periódico na internet].2002 [acessado 2011 ago 1]; 2(2):[cerca de 10 p]. Disponível em: http://www.scielo.br/pdf/rbsmi/v2n2/17109.pdf.

2. Souza EC, Martinez, MB, Taddei, CR, Mukai, L, Gilio, AE, Racz, ML, Silva, L, Ejzenberg, B, Okay, Y. Perfil etiológico das diarreias agudas de crianças atendidas em São Paulo. J Pediatr. [periódico na internet]. 2002 [acessado 2011 jun 1]; 78(1): [cerca de 08 p]. Disponível em: http://www.scielo.br/pdf/jped/v78n1/ v78n1a08.pdf

3. Schnack FJ, Fontana, LM, Barbosa PR, Silva LSM, Baillargeon CMM, Barichello T, Póvoa MM, Cavasini CE, Machado RLD. Enteropatógenos associados com diarréia infantil $(<5$ anos de idade $)$ em amostra da população da área metropolitana de Criciúma, Santa Catarina, Brasil. Cad Saude Publica 2003; 19(4):12051208.

4. Cauás RC, Falbo AR, Correia JB, Oliveira, KMM, Montenegro, FMU. Diarréia por rotavírus em crianças desnutridas hospitalizadas no Instituto Materno Infantil Prof. Fernando Figueira, IMIP. Rev. Bras. Saude Mater Infant. [periódico na internet]. 2006 Maio [acessado 2011 jul 10]; 6(1): [cerca de 7 p]. Disponível em: http:// www.scielo.br/pdf/rbsmi/v6s1/30508.pdf

5. Assis AMO, Barreto ML, Santos NS, Oliveira LPM, Santos SMC, Pinheiro SMC. Desigualdade, pobreza e condições de saúde e nutrição na infância no Nordeste brasileiro. Cad Saude Publica 2007; 23(10):2337-2350.

6. Oliveira CSM, Cardoso MA, Araújo TS, Muniz PT. Anemia em crianças de 6 a 59 meses e fatores associados no Município de Jordão, Estado do Acre, Brasil. Cad Saude Publica 2011; 27(5):1008-1020.

7. Vanderlei LCM, Silva GAP, Braga JU. Fatores de risco para internamento por diarréia aguda em menores de dois anos: estudo de caso-controle. Cad Saude Publica 2013; 19(2):455-463.

8. Vanderlei LCM, Silva GAP. Diarreia Aguda: o conhecimento materno sobre a doença reduz o número de hospitalizações nos menores de dois anos? Rer. Assoc. Med. Bras. [periódico na internet]. 2004 [acessado 2011 ago 02]; 50(3): [cerca de 06 p]. Disponível em: http:// www.scielo.br/pdf/ramb/v50n3/21659.pdf

9. Teixeira JC, Heller L. Fatores ambientais associados à diarréia infantil em áreas de assentamento subnormal em Juiz de Fora, Minas Gerais. Rev. Bras. Saude Mater. Infant. [periódico na internet]. 2005 [acessado 2011 ago 02]; 5(4): [cerca de 06 p]. Disponível em: http:// www.scielo.br/scielo.php?script=sci_arttext\&pid= S1519-38292005000400008

10. Joventino ES, Silva SF, Rogerio RF, Freitas GL, Ximenes LB, Moura ERF. Comportamento da diarreia infantil antes e após o consumo de água pluvial em município do semiárido brasileiro. Texto Contexto Enfermagem. [periódico na internet]. 2010 [acessado 2011 ago 02]; 19(4): [cerca de 09 p]. Disponível em: http://www.scielo. $\mathrm{br} / \mathrm{pdf} / \mathrm{tce} / \mathrm{v} 19 \mathrm{n} 4 / 12 . \mathrm{pdf}$
11. Dias DM, Silva AP, Helfer AM, Maciel AMTR, Loureiro ECB, Souza COS. Morbimortalidade por gastroenterites no Estado do Pará, Brasil. Rer. Pan-Amaz Saude. [periódico na internet]. 2010 [acessado 2011 jul 18]; 1(1): [cerca de 08 p]. Disponível em: http:// scielo.iec.pa.gov.br/scielo.php?script=sci_arttext\&pid $=$ S2176-62232010000100008\&lng $=$ pt\&nrm $=$ iss

12. Black RE, Morris SS, Bryce J. Where and why are 10 million children dying every year?. The Lancet 2003; 361(1):2226-2234.

13. World Health Organization (WHO). The World health report: 2003: shaping the future. In: World Health Organization (WHO). Global Health: today's challenges, Chapter 1. Geneva: WHO; 2003. p. 3-22.

14. Queiroz JTM, Heller L, Silva SR. Análise da Correlação de Ocorrência da Doença Diarreica Aguda com a Qualidade da Água para Consumo Humano no Município de Vitória - ES. Saúde e Sociedade 2009; 18(3):479-489.

15. Instituto Brasileiro de Geografia e Estatística (IBGE). Censo Demográfico 2010. Resultados Preliminares do Universo. Rio de Janeiro: IBGE; 2010.

16. Paim J, Travassos C, Almeida C, Bahia L, Macinko J. O sistema de saúde brasileiro: história, avanços e desafios. The Lancet 2011; 1:11-31.

17. Rede Interagencial de Informações para a Saúde. Indicadores Básicos de saúde no Brasil: conceitos e aplicações. Brasília: Organização Pan-Americana de Saúde; 2009.

18. Brasil. Ministério da Saúde (MS). Departamento de Informática do SUS (DATASUS). Sistema de Informação de Mortalidade. Mortalidade - Brasil. Óbito por residência por Capítulo CID 10 segundo região. Brasília: MS; 2011.

19. Bühler HF. A diarreia infantil no Brasil: análise espacial de indicadores integrados de saúde e ambiente, 2010 [dissertação]. Cáceres: Universidade do Estado de Mato Grosso; 2010

20. Fischer TK, Lima D, Rosa R, Osório D, Boing AF. A mortalidade infantil no Brasil: série histórica entre 1994-2004 e associação com indicadores socioeconômicos em municípios de médio e grande porte. Medicina (Ribeirão Preto). [periódico na internet]. 2007 [acessado 2013 jan 19]; 40(4): [cerca de 08 p]. Disponível em: http://www.fmrp.usp.br/revista/2007/vol40n4/aol _mortalidade_infantil_brasil_tendencia_desigualdades. pdf

21. Costa SS, Héller L, Brandão CCS, Colosimo EA. Indicadores epidemiológicos aplicáveis a estudos sobre a associação entre saneamento e saúde de base municipal. Eng. Sanit. Ambient [periódico na internet]. 2005 [acessado 2012 out 10]; 10(2): [cerca de 10 p]. Disponível em: http://www.scielo.br/pdf/esa/v10n2/a05v10n2.pdf

22. Kronemberger DMP, Clevelário Júnior J. Análise dos impactos na saúde e no Sistema Único de Saúde decorrente de agravos relacionados ao esgotamento sanitário inadequado nos municípios brasileiros com mais de 300.000 habitantes. 2010. São Paulo: Instituto Trata Brasil; 2010. 
23. Oliveira TCR, Latorre MRDO. Tendências da internação e da mortalidade infantil por diarréia: Brasil, 1995 a 2005. Rev Saude Publica 2010; 44(1):102-111.

24. Brasil. Ministério da Saúde (MS). Inquérito Nacional de Saúde e Nutrição dos Povos Indígenas. Relatório Final. Rio de Janeiro: MS; 2009.

25. Marinho GL, Santos RV, Pereira NOM. Classificação dos domicílios "indígenas" no Censo Demográfico 2000: subsídios para a análise de condições de saúde. R. bras. Est. Pop. [periódico na internet]. 2011 jul-dez [acessado 2012 out 20]; 28(2): [cerca de 18 p]. Disponível em: http://www.scielo.br/pdf/rsp/v44n1/11.pdf

26. Visser S, Giatti LL, Carvalho RAC, Guerreiro JCH. Estudo da associação entre fatores socioambientais e prevalência de parasitose intestinal em área periférica da cidade de Manaus (AM, Brasil). Cien Saude Colet 2011; 16(8):3481-3492.

27. Mato Grosso. Secretaria Estadual de Saúde. Plano Diretor de Regionalização. 2005.

28. Vasconcelos MJOB, Batista Filho M. Doenças diarreicas em menores de cinco anos no Estado de Pernambuco: prevalência e utilização de serviços de saúde. Rev. bras. epidemiol. [periódico na internet]. 2008 [acessado 2012 nov 10]; 11(1): [cerca de 10 p]. Disponível em: http://www.scielo.br/pdf/rbepid/v11n1/12.pdf

29. Fonseca FR, Vasconcelos CH. Análise espacial das Doenças Relacionadas ao Saneamento Ambiental Inadequado no Brasil. Cad Saúde Colet [periódico na internet]. 2011 [acessado 2012 nov 10]; 19(4): [cerca de 05 p]. Disponível em: http://www.cadernos.iesc.ufrj.br/ cadernos/images/csc/2011_4/artigos/csc_v19n4_448453.pdf

30. Calijuri ML, Santiago AF, Camargo RA, Moreira Neto RF. Estudo de indicadores de saúde ambiental e de saneamento em cidade do Norte do Brasil. Revista de Engenharia Sanitária e Ambiental. [periódico na internet]. 2009 [acessado 2012 out 10]; 14(1): [cerca de 09 p]. Disponível em: http://www.scielo.br/pdf/esa/v14n1/ v14n1a03.pdf
31. Fonseca EOL, Teixeira MG, Barreto ML, Carmo EH, Costa MCN. Prevalência e fatores associados às geo -helmintiases em crianças residentes em municípios com baixo IDH no Norte e Nordeste brasileiros. Cad Saude Publica 2010; 26(1):143-152.

32. Franco Netto G, Freitas CM, Andahur JP, Pedroso MM, Rohlfs DB. Impactos socioambientais na situação de saúde da população brasileira: Estudo de indicadores relacionados ao saneamento ambiental inadequado. Rev. Tempus Actas Saúde [periódico na internet]. 2009 [acessado 2011 ago 10]; 4(4): [cerca de 19 p]. Disponível em: http://www.tempusactas.unb.br/index.php/ tempus/article/viewFile/745/754

33. Brasil. Ministério do Desenvolvimento Social e Combate à Fome. Cadernos de Estudos Desenvolvimento Social em Debate. Brasília: Ministério do Desenvolvimento Social e Combate à Fome; 2008.

34. Juliano EFGA, Feuerwerker LCM, Coutinho SMV, Malheiros TF. Racionalidade e saberes para a universalização do saneamento em áreas de vulnerabilidade social. Cien Saude Colet 2012; 17(11):3037-3046.

Artigo apresentado em 06/07/2014

Aprovado em 11/07/2014

Versão final apresentada em 12/07/2014 